\title{
On some inequalities in 2-metric spaces
}

Mohamed Jleli ${ }^{1}$ and Bessem Samet ${ }^{1 *}$ (D)

${ }^{*}$ Correspondence:

bsamet@ksu.edu.sa

${ }^{1}$ Department of Mathematics,

College of Science, King Saud

University, P.O. Box 2455, Riyadh,

11451, Saudi Arabia

\section{Springer}

\begin{abstract}
In this paper, we establish new inequalities in the setting of 2-metric spaces and provide their geometric interpretations. Some of our results are extensions of those obtained by Dragomir and Goşa (J. Indones. Math. Soc. 11(1):33-38, 2005) in the setting of metric spaces.
\end{abstract}

Keywords: 2-metric spaces; 2-normed linear spaces; Metric inequalities

\section{Introduction and preliminaries}

We start this section by recalling an interesting metric-type inequality due to Dragomir and Goşa [7]. Let us first fix some notations. We denote by $\mathbb{N}$ the set of positive natural numbers, that is, $\mathbb{N}=\{1,2, \ldots\}$. For $n \in \mathbb{N}$, let

$$
\Pi_{n}=\left\{\left(p_{1}, p_{2}, \ldots, p_{n}\right) \in \mathbb{R}^{n}: p_{i} \geq 0(i=1,2, \ldots, n), \sum_{i=1}^{n} p_{i}=1\right\} .
$$

Theorem 1.1 (Dragomir-Goșa [7]) Let $(X, d)$ be a metric space. Then, for all $n \in \mathbb{N}, n \geq 2$, $\left(p_{1}, p_{2}, \ldots, p_{n}\right) \in \Pi_{n}$, and $\left\{x_{i}\right\}_{i=1}^{n} \subset X$,

$$
\sum_{i=1}^{n-1} \sum_{j=i+1}^{n} p_{i} p_{j} d\left(x_{i}, x_{j}\right) \leq \inf _{x \in X} \sum_{i=1}^{n} p_{i} d\left(x_{i}, x\right) .
$$

Moreover, the inequality is optimal in the sense that the multiplicative coefficient $C=1$ on the right-hand side of (1.1) (in front of inf) cannot be replaced by a smaller real number.

In the particular case where $p_{i}=\frac{1}{n}(i=1,2, \ldots, n),(1.1)$ reduces to

$$
\sum_{i=1}^{n-1} \sum_{j=i+1}^{n} d\left(x_{i}, x_{j}\right) \leq n \inf _{x \in X} \sum_{i=1}^{n} d\left(x_{i}, x\right) .
$$

This inequality can be interpreted as follows. Let $P$ be a polygon in a metric space with $n$ vertices, and let $x$ be an arbitrary point in the space. Then the sum of all edges and diagonals of $P$ is less than $n$ times the sum of the distances from $x$ to the vertices of $P$.

(c) The Author(s) 2021. This article is licensed under a Creative Commons Attribution 4.0 International License, which permits use, sharing, adaptation, distribution and reproduction in any medium or format, as long as you give appropriate credit to the original author(s) and the source, provide a link to the Creative Commons licence, and indicate if changes were made. The images or other third party material in this article are included in the article's Creative Commons licence, unless indicated otherwise in a credit line to the material. If material is not included in the article's Creative Commons licence and your intended use is not permitted by statutory regulation or exceeds the permitted use, you will need to obtain permission directly from the copyright holder. To view a copy of this licence, visit http://creativecommons.org/licenses/by/4.0/. 
In the same reference [7] the authors provided some interesting applications of inequality (1.1) to normed linear spaces and pre-Hilbert spaces. For more results on metric inequalities, we refer to $[1,6,12]$ and the references therein.

In this paper, we derive new inequalities in 2-metric spaces and 2-normed linear spaces. In particular, we obtain an extension of Theorem 1.1 to the setting of 2-metric spaces and provide a geometric interpretation of the obtained inequality.

Before stating and proving our results, let us recall briefly some basic notions related to 2-metric spaces and 2-normed linear spaces.

In 1963, Gähler [10] introduced the notion of 2-metric spaces as follows. Let $X$ be a nonempty set, and let $D: X \times X \times X \rightarrow \mathbb{R}$. We say that $D$ is a 2-metric on $X$ if the following conditions are satisfied:

$\left(D_{1}\right)$ for all $x, y \in X$ with $x \neq y$, there exists $z=z(x, y) \in X$ such that

$$
D(x, y, z) \neq 0
$$

$\left(D_{2}\right) D(x, y, z)=0$ when at least two elements of $\{x, y, z\} \subset X$ are equal;

$\left(D_{3}\right)$ for all $x, y, z \in X$,

$$
D(x, y, z)=D(x, z, y)=D(y, z, x)
$$

$\left(D_{4}\right)$ for all $x, y, z, u \in X$,

$$
D(x, y, z) \leq D(u, y, z)+D(x, u, z)+D(x, y, u) .
$$

In this case, the pair $(X, D)$ is called a 2-metric space.

Let us mention some remarks following from properties $\left(D_{1}\right)-\left(D_{4}\right)$.

- Given $x, y, z \in X$, we denote by $\sigma(x, y, z)$ any permutation of the elements $x, y$, and $z$.

By $\left(D_{3}\right)$ we deduce that

$$
D(x, y, z)=D(\sigma(x, y, z)), \quad x, y, z \in X
$$

- Let $x, y, z \in X$. By $\left(D_{3}\right)$ and $\left(D_{4}\right)$, for all $u \in X$, we have

$$
\begin{aligned}
D(x, y, z) \\
\quad \leq D(u, y, z)+D(x, u, z)+D(x, y, u) \\
\quad \leq D(x, y, z)+D(u, x, z)+D(u, y, x)+D(x, u, z)+D(x, y, u) \\
\quad=D(x, y, z)+2 D(u, x, z)+2 D(u, y, x),
\end{aligned}
$$

which yields

$$
D(u, x, z)+D(u, y, x) \geq 0 .
$$

Taking $u=y$ in this inequality and using $\left(D_{2}\right)$, we obtain

$$
D(x, y, z) \geq 0, \quad x, y, z \in X
$$


Example 1.1 (see [10]) Let $D: \mathbb{R}^{N} \times \mathbb{R}^{N} \times \mathbb{R}^{N} \rightarrow \mathbb{R}, N \in \mathbb{N}, N \geq 2$, be the mapping defined by

$$
D\left(A_{1}, A_{2}, A_{3}\right)=\frac{1}{2}\left\|\overrightarrow{A_{1} A_{2}} \times \overrightarrow{A_{1} A_{3}}\right\|_{2}, \quad A_{1}, A_{2}, A_{3} \in \mathbb{R}^{N},
$$

where $\times$ denotes the cross product in $\mathbb{R}^{N}$, and $\|\cdot\|_{2}$ denotes the Euclidean norm in $\mathbb{R}^{N}$. Then $D$ is a 2-metric on $X=\mathbb{R}^{N}$. Note that $D\left(A_{1}, A_{2}, A_{3}\right)$ is equal to the area of the triangle spanned by $A_{1}, A_{2}$, and $A_{3}$.

In the same reference [10], Gähler introduced the notion of 2-normed linear spaces as follows. Let $X$ be a linear space over $\mathbb{R}$ of dimension $1<L \leq \infty$. Let $\|\cdot, \cdot\|: X \times X \rightarrow \mathbb{R}$ be a given mapping. We say that $\|\cdot, \cdot\|$ is a 2-norm on $X$ if the following conditions are satisfied for all $x, y, z \in X$ and $\lambda \in \mathbb{R}$ :

$\left(N_{1}\right)\|x, y\|=0$ if and only if $x$ and $y$ are linearly dependent;

$\left(N_{2}\right)\|x, y\|=\|y, x\|$;

$\left(N_{3}\right)\|\lambda x, y\|=|\lambda|\|x, y\|$;

$\left(N_{4}\right)\|x, y+z\| \leq\|x, y\|+\|x, z\|$.

In this case, the pair $(X,\|\cdot, \cdot\|)$ is said to be a 2-normed space.

We now give some remarks following from $\left(N_{1}\right)-\left(N_{4}\right)$ :

- By $\left(N_{2}\right)$ and $\left(N_{3}\right)$, for all $x, y \in X$ and $\lambda, \mu \in \mathbb{R}$, we have

$$
\|\lambda x, \mu y\|=|\lambda||\mu|\|x, y\|=\|\mu x, \lambda y\| .
$$

- If $\|\cdot, \cdot\|$ is a 2-norm on $X$, then the mapping $D: X \times X \times X \rightarrow \mathbb{R}$ defined by

$$
D(x, y, z)=\|x-z, y-z\|, \quad x, y, z \in X
$$

is a 2-metric on $X$. Note that if $L=1$, then condition $\left(D_{1}\right)$ is not satisfied by $D$. Namely, by $\left(N_{1}\right)$, if $X=\operatorname{span}\{a\}, a \in X$, then for all $x, y, z \in X$, there exist $\lambda, \mu, \gamma \in \mathbb{R}$ such that

$$
D(x, y, z)=D(\lambda a, \mu a, \gamma a)=\|(\lambda-\gamma) a,(\mu-\gamma) a\|=|(\lambda-\gamma)(\mu-\gamma)|\|a, a\|=0 .
$$

- From the above remark and the positivity of $D$ we deduce that

$$
\|x, y\| \geq 0, \quad x, y \in X
$$

- Let $x, y, z \in X$ and $\lambda_{1}, \lambda_{2} \in \mathbb{R}$. By $\left(N_{2}\right)$ and $\left(N_{4}\right)$ we have

$$
\begin{aligned}
\left\|\lambda_{1} x+\lambda_{2} y, z\right\| & =\left\|z, \lambda_{1} x+\lambda_{2} y\right\| \\
& \leq\left\|z, \lambda_{1} x\right\|+\left\|z, \lambda_{2} y\right\| \\
& =\left|\lambda_{1}\right|\|x, z\|+\left|\lambda_{2}\right|\|y, z\| .
\end{aligned}
$$

Hence by induction we deduce that if $x_{i}, z \in X$ and $\lambda_{i} \in \mathbb{R}, i=1,2, \ldots, m$, then

$$
\left\|\lambda_{1} x_{1}+\lambda_{2} x_{2}+\cdots+\lambda_{m} x_{m}, z\right\| \leq \sum_{i=1}^{m}\left|\lambda_{i}\right|\left\|x_{i}, z\right\| .
$$


For more details about 2-metric spaces and 2-normed linear spaces, see, for example, $[2-5,8,9,11,13-17]$ and the references therein.

\section{Results and proofs}

In this section, we state and prove our main results and provide some interesting consequences.

Theorem 2.1 Let $(X, D)$ be a 2-metric space. Then, for all $n \in \mathbb{N}, n \geq 3,\left(p_{1}, p_{2}, \ldots, p_{n}\right) \in$ $\Pi_{n}$, and $\left\{x_{i}\right\}_{i=1}^{n} \subset X$,

$$
\sum_{i=1}^{n-2} \sum_{j=i+1}^{n-1} \sum_{k=j+1}^{n} p_{i} p_{j} p_{k} D\left(x_{i}, x_{j}, x_{k}\right) \leq \inf _{x \in X} \sum_{i=1}^{n-1} \sum_{j=i+1}^{n} p_{i} p_{j} D\left(x, x_{i}, x_{j}\right)
$$

Moreover, the inequality is optimal in the sense that the multiplicative coefficient $C=1$ on the right-hand side of (2.1) (in front of inf) cannot be replaced by a smaller real number.

Proof Let $n \in \mathbb{N}, n \geq 3,\left(p_{1}, p_{2}, \ldots, p_{n}\right) \in \Pi_{n}$, and $\left\{x_{i}\right\}_{i=1}^{n} \subset X$. Let $x$ be an arbitrary element of $X$. For all $i, j, k \in\{1,2, \ldots, n\}$, we have

$$
D\left(x_{i}, x_{j}, x_{k}\right) \leq D\left(x, x_{j}, x_{k}\right)+D\left(x_{i}, x, x_{k}\right)+D\left(x_{i}, x_{j}, x\right) .
$$

Multiplying this inequality by $p_{i} p_{j} p_{k}$ and taking the sum from 1 to $n$, we obtain

$$
\sum_{i=1}^{n} \sum_{j=1}^{n} \sum_{k=1}^{n} p_{i} p_{j} p_{k} D\left(x_{i}, x_{j}, x_{k}\right) \leq A+B+C
$$

where

$$
A=\sum_{i=1}^{n} \sum_{j=1}^{n} \sum_{k=1}^{n} p_{i} p_{j} p_{k} D\left(x, x_{j}, x_{k}\right), \quad B=\sum_{i=1}^{n} \sum_{j=1}^{n} \sum_{k=1}^{n} p_{i} p_{j} p_{k} D\left(x_{i}, x, x_{k}\right)
$$

and

$$
C=\sum_{i=1}^{n} \sum_{j=1}^{n} \sum_{k=1}^{n} p_{i} p_{j} p_{k} D\left(x_{i}, x_{j}, x\right)
$$

Sine $\sum_{i=1}^{n} p_{i}=1$, by the symmetry of $D$ we deduce that

$$
A=B=C=\sum_{i=1}^{n} \sum_{j=1}^{n} p_{i} p_{j} D\left(x, x_{i}, x_{j}\right)
$$

On the other hand, by $\left(D_{2}\right)-\left(D_{3}\right)$ we have

$$
\begin{aligned}
\sum_{i=1}^{n} \sum_{j=1}^{n} p_{i} p_{j} D\left(x, x_{i}, x_{j}\right) & =\sum_{i<j} p_{i} p_{j} D\left(x, x_{i}, x_{j}\right)+\sum_{j<i} p_{i} p_{j} D\left(x, x_{i}, x_{j}\right) \\
& =2 \sum_{i<j} p_{i} p_{j} D\left(x, x_{i}, x_{j}\right)
\end{aligned}
$$


that is,

$$
\sum_{i=1}^{n} \sum_{j=1}^{n} p_{i} p_{j} D\left(x, x_{i}, x_{j}\right)=2 \sum_{i=1}^{n-1} \sum_{j=i+1}^{n} p_{i} p_{j} D\left(x, x_{i}, x_{j}\right) .
$$

Similarly, we have

$$
\begin{aligned}
\sum_{i=1}^{n} & \sum_{j=1}^{n} \sum_{k=1}^{n} p_{i} p_{j} p_{k} D\left(x_{i}, x_{j}, x_{k}\right) \\
= & \sum_{i<j<k} p_{i} p_{j} p_{k} D\left(x_{i}, x_{j}, x_{k}\right)+\sum_{i<k<j} p_{i} p_{j} p_{k} D\left(x_{i}, x_{j}, x_{k}\right)+\sum_{j<i<k} p_{i} p_{j} p_{k} D\left(x_{i}, x_{j}, x_{k}\right) \\
& +\sum_{j<k<i} p_{i} p_{j} p_{k} D\left(x_{i}, x_{j}, x_{k}\right)+\sum_{k<i<j} p_{i} p_{j} p_{k} D\left(x_{i}, x_{j}, x_{k}\right)+\sum_{k<j<i} p_{i} p_{j} p_{k} D\left(x_{i}, x_{j}, x_{k}\right) \\
= & 6 \sum_{i<j<k} p_{i} p_{j} p_{k} D\left(x_{i}, x_{j}, x_{k}\right),
\end{aligned}
$$

that is,

$$
\sum_{i=1}^{n} \sum_{j=1}^{n} \sum_{k=1}^{n} p_{i} p_{j} p_{k} D\left(x_{i}, x_{j}, x_{k}\right)=6 \sum_{i=1}^{n-2} \sum_{j=i+1}^{n-1} \sum_{k=j+1}^{n} p_{i} p_{j} p_{k} D\left(x_{i}, x_{j}, x_{k}\right) .
$$

Hence, using (2.2), (2.3), (2.4), and (2.5), we obtain

$$
\sum_{i=1}^{n-2} \sum_{j=i+1}^{n-1} \sum_{k=j+1}^{n} p_{i} p_{j} p_{k} D\left(x_{i}, x_{j}, x_{k}\right) \leq \sum_{i=1}^{n-1} \sum_{j=i+1}^{n} p_{i} p_{j} D\left(x, x_{i}, x_{j}\right) .
$$

Since this inequality holds for all $x \in X$, we deduce (2.1).

Suppose now that there exists a constant $C>0$ such that

$$
\sum_{i=1}^{n-2} \sum_{j=i+1}^{n-1} \sum_{k=j+1}^{n} p_{i} p_{j} p_{k} D\left(x_{i}, x_{j}, x_{k}\right) \leq C \inf _{x \in X} \sum_{i=1}^{n-1} \sum_{j=i+1}^{n} p_{i} p_{j} D\left(x, x_{i}, x_{j}\right)
$$

for all $n \in \mathbb{N}, n \geq 3,\left(p_{1}, p_{2}, \ldots, p_{n}\right) \in \Pi_{n}$, and $\left\{x_{i}\right\}_{i=1}^{n} \subset X$. Taking $n=3$ in (2.6), we obtain

$$
p_{1} p_{2} p_{3} D\left(x_{1}, x_{2}, x_{3}\right) \leq C\left[p_{1} p_{2} D\left(x, x_{1}, x_{2}\right)+p_{1} p_{3} D\left(x, x_{1}, x_{3}\right)+p_{2} p_{3} D\left(x, x_{2}, x_{3}\right)\right]
$$

for all $\left(p_{1}, p_{2}, p_{3}\right) \in \Pi_{3},\left\{x_{i}\right\}_{i=1}^{3} \subset X$, and $x \in X$. In particular, for $x=x_{1}$ and $\left(p_{1}, p_{2}, p_{3}\right)=$ $(2 \varepsilon-1,1-\varepsilon, 1-\varepsilon), \frac{1}{2}<\varepsilon<1$, by $\left(D_{2}\right)$ we obtain

$$
(2 \varepsilon-1)(1-\varepsilon)^{2} D\left(x_{1}, x_{2}, x_{3}\right) \leq C(1-\varepsilon)^{2} D\left(x_{1}, x_{2}, x_{3}\right),
$$

which yields

$$
2 \varepsilon-1 \leq C, \quad \frac{1}{2}<\varepsilon<1 .
$$

Passing to the limit as $\varepsilon \rightarrow 1^{-}$, we get that $C \geq 1$, which proves the sharpness of (2.1). 
Corollary 2.1 Let $(X, D)$ be a 2-metric space. Then, for all $n \in \mathbb{N}, n \geq 3$, and $\left\{x_{i}\right\}_{i=1}^{n} \subset X$,

$$
\sum_{i=1}^{n-2} \sum_{j=i+1}^{n-1} \sum_{k=j+1}^{n} D\left(x_{i}, x_{j}, x_{k}\right) \leq n \inf _{x \in X} \sum_{i=1}^{n-1} \sum_{j=i+1}^{n} D\left(x, x_{i}, x_{j}\right)
$$

Proof By (2.1) with

$$
p_{i}=\frac{1}{n}, \quad i \in\{1,2, \ldots, n\}
$$

(2.7) follows.

Corollary 2.1 has the following geometric interpretation.

Corollary 2.2 Let $n \in \mathbb{N}, n \geq 3$, and let $A_{1}, A_{2}, \ldots, A_{n}, A$ be $n+1$ points of $\mathbb{R}^{N}, N \geq 2$. Then the sum of the areas of all triangles with vertices belonging to the set of points $\left\{A_{i}\right.$ : $i=1,2, \ldots, n\}$ is less than $n$ times the sum of the areas of all triangles such that one of the vertices is the point $A$ and the other vertices belong to the set of points $\left\{A_{i}: i=1,2, \ldots, n\right\}$.

Proof The result follows immediately from Corollary 2.1 by taking $X=\mathbb{R}^{N}$ and $D$, the 2-metric defined by (1.2).

Corollary 2.3 Let $(X, D)$ be a 2-metric space, $n \in \mathbb{N}, n \geq 3,\left(p_{1}, p_{2}, \ldots, p_{n}\right) \in \Pi_{n}$, and $\left\{x_{i}\right\}_{i=1}^{n} \subset X$. Let $x \in X$ be such that

$$
D\left(x, x_{i}, x_{j}\right) \leq r, \quad i, j \in\{1,2, \ldots, n\}
$$

for some $r>0$. Then

$$
\sum_{i=1}^{n-2} \sum_{j=i+1}^{n-1} \sum_{k=j+1}^{n} p_{i} p_{j} p_{k} D\left(x_{i}, x_{j}, x_{k}\right) \leq\left(\sum_{i=1}^{n-1} \sum_{j=i+1}^{n} p_{i} p_{j}\right) r
$$

Proof By (2.1) we have

$$
\sum_{i=1}^{n-2} \sum_{j=i+1}^{n-1} \sum_{k=j+1}^{n} p_{i} p_{j} p_{k} D\left(x_{i}, x_{j}, x_{k}\right) \leq \sum_{i=1}^{n-1} \sum_{j=i+1}^{n} p_{i} p_{j} D\left(x, x_{i}, x_{j}\right) .
$$

On the other hand, using (2.8), we obtain

$$
\sum_{i=1}^{n-1} \sum_{j=i+1}^{n} p_{i} p_{j} D\left(x, x_{i}, x_{j}\right) \leq r \sum_{i=1}^{n-1} \sum_{j=i+1}^{n} p_{i} p_{j}
$$

Combining (2.10) with (2.11), (2.9) follows.

Corollary 2.4 Let $X$ be a linear space over $\mathbb{R}$ of dimension $1<L \leq \infty$, and let $\|\cdot, \cdot\|$ be a 2-norm on $X$. Then, for all $n \in \mathbb{N}, n \geq 3,\left(p_{1}, p_{2}, \ldots, p_{n}\right) \in \Pi_{n}$, and $\left\{x_{i}\right\}_{i=1}^{n} \subset X$,

$$
\sum_{i=1}^{n-2} \sum_{j=i+1}^{n-1} \sum_{k=j+1}^{n} p_{i} p_{j} p_{k}\left\|x_{i}-x_{k}, x_{j}-x_{k}\right\| \leq \inf _{x \in X} \sum_{i=1}^{n-1} \sum_{j=i+1}^{n} p_{i} p_{j}\left\|x-x_{j}, x_{i}-x_{j}\right\| .
$$


Moreover, the inequality is optimal in the sense that the multiplicative coefficient $C=1$ on the right-hand side of (2.12) (in front of inf) cannot be replaced by a smaller real number.

Proof Consider the 2-metric $D$ on $X$ defined by (1.3). Then (2.12) follows by (2.1).

Theorem 2.2 Let $X$ be a linear space over $\mathbb{R}$ of dimension $1<L \leq \infty$, and let $\|\cdot \cdot\|$ be a 2-norm on $X$. Then, for all $n \in \mathbb{N}, n \geq 3,\left(p_{1}, p_{2}, \ldots, p_{n}\right) \in \Pi_{n}$, and $\left\{x_{i}\right\}_{i=1}^{n} \subset X$,

$$
\frac{1}{6} \sum_{i=1}^{n} \sum_{j=1}^{n} p_{i} p_{j}\left\|x_{p}-x_{i}, x_{j}-x_{i}\right\| \leq \rho_{n} \leq \sum_{i=1}^{n-1} \sum_{j=i+1}^{n} p_{i} p_{j}\left\|x_{p}-x_{j}, x_{i}-x_{j}\right\|,
$$

where

$$
\rho_{n}=\sum_{i=1}^{n-2} \sum_{j=i+1}^{n-1} \sum_{k=j+1}^{n} p_{i} p_{j} p_{k}\left\|x_{i}-x_{k}, x_{j}-x_{k}\right\|, \quad x_{p}=\sum_{i=1}^{n} p_{i} x_{i} .
$$

Proof Using (2.12) with $x=x_{p}$, we obtain

$$
\rho_{n} \leq \sum_{i=1}^{n-1} \sum_{j=i+1}^{n} p_{i} p_{j}\left\|x_{p}-x_{j}, x_{i}-x_{j}\right\|
$$

By (2.5) we have

$$
\rho_{n}=\frac{1}{6} \sum_{i=1}^{n} \sum_{j=1}^{n} \sum_{k=1}^{n} p_{i} p_{j} p_{k}\left\|x_{i}-x_{k}, x_{j}-x_{k}\right\|
$$

On the other hand, using $\left(N_{2}\right)$, we obtain

$$
\sum_{i=1}^{n} \sum_{j=1}^{n} \sum_{k=1}^{n} p_{i} p_{j} p_{k}\left\|x_{i}-x_{k}, x_{j}-x_{k}\right\|=\sum_{k=1}^{n} \sum_{i=1}^{n} p_{k} p_{i} \sum_{j=1}^{n}\left\|p_{j}\left(x_{j}-x_{k}\right), x_{i}-x_{k}\right\| .
$$

Next, by (1.4) we have that

$$
\begin{aligned}
\sum_{j=1}^{n}\left\|p_{j}\left(x_{j}-x_{k}\right), x_{i}-x_{k}\right\| & \geq\left\|\sum_{j=1}^{n} p_{j}\left(x_{j}-x_{k}\right), x_{i}-x_{k}\right\| \\
& =\left\|x_{p}-x_{k}, x_{i}-x_{k}\right\| .
\end{aligned}
$$

Hence it follows from (2.15), (2.16), and (2.17) that

$$
\rho_{n} \geq \frac{1}{6} \sum_{k=1}^{n} \sum_{i=1}^{n} p_{k} p_{i}\left\|x_{p}-x_{k}, x_{i}-x_{k}\right\|=\frac{1}{6} \sum_{i=1}^{n} \sum_{j=1}^{n} p_{i} p_{j}\left\|x_{p}-x_{i}, x_{j}-x_{i}\right\| .
$$

Finally, (2.13) follows from (2.14) and (2.18).

For our next result, we need some notations.

Given three points $A, B, C \in \mathbb{R}^{N}, N \geq 2$, we denote by $\triangle(A, B, C)$ the area of the triangle with vertices $A, B$, and $C$. 
Let $n \in \mathbb{N}, n \geq 3$. For $n$ points $A_{1}, A_{2}, \ldots, A_{n} \in \mathbb{R}^{N}$, let

$$
\mathcal{S}\left(A_{1}, A_{2}, \ldots, A_{n}\right)=\sum_{i=1}^{n} \triangle\left(A_{i}, A_{i+1}, A_{i+2}\right), \quad A_{n+1}=A_{1}, \quad A_{n+2}=A_{2} .
$$

We introduce the set

$$
\Lambda_{n}=\left\{\left\{A_{1}, A_{2}, \ldots, A_{n}\right\} \subset \mathbb{R}^{N}: \mathcal{S}\left(A_{1}, A_{2}, \ldots, A_{n}\right)=1\right\}
$$

and the quantity

$$
\alpha_{n}=\inf _{\left\{A_{1}, A_{2}, \ldots, A_{n}\right\} \in \Lambda_{n}} \sum_{i=1}^{n-2} \sum_{j=i+1}^{n-1} \sum_{k=j+1}^{n} \triangle\left(A_{i}, A_{j}, A_{k}\right)
$$

Theorem 2.3 For all $n \in \mathbb{N}, n \geq 3$, we have that $\alpha_{n} \geq \frac{n}{18}$.

Proof First, for all $A, B, C \in \mathbb{R}^{N}$, we have

$$
\triangle(A, B, C)=D(A, B, C),
$$

where $D$ is the 2-metric defined by (1.2). On the other hand, given $\left\{A_{1}, A_{2}, \ldots, A_{n}\right\} \in \Lambda_{n}$, for all $j \in\{1,2, \ldots, n\}$, by $\left(D_{4}\right)$, we have

$$
D\left(A_{j}, A_{j+1}, A_{j+2}\right) \leq D\left(P, A_{j+1}, A_{j+2}\right)+D\left(A_{j}, P, A_{j+2}\right)+D\left(A_{j}, A_{j+1}, P\right)
$$

for all $P \in\left\{A_{1}, A_{2}, \ldots, A_{n}\right\}$. Taking the sum over $j$ from 1 to $n$, we get that

$$
\mathcal{S}\left(A_{1}, A_{2}, \ldots, A_{n}\right) \leq \sum_{j=1}^{n} D\left(P, A_{j+1}, A_{j+2}\right)+\sum_{j=1}^{n} D\left(A_{j}, P, A_{j+2}\right)+\sum_{j=1}^{n} D\left(A_{j}, A_{j+1}, P\right)
$$

that is,

$$
1 \leq \sum_{j=1}^{n} D\left(P, A_{j+1}, A_{j+2}\right)+\sum_{j=1}^{n} D\left(A_{j}, P, A_{j+2}\right)+\sum_{j=1}^{n} D\left(A_{j}, A_{j+1}, P\right) .
$$

Notice that

$$
\begin{aligned}
\sum_{j=1}^{n} D\left(P, A_{j+1}, A_{j+2}\right) & =\sum_{j=2}^{n+1} D\left(P, A_{j}, A_{j+1}\right) \\
& =\sum_{j=1}^{n} D\left(P, A_{j}, A_{j+1}\right)-D\left(P, A_{1}, A_{2}\right)+D\left(P, A_{n+1}, A_{n+2}\right) \\
& =\sum_{j=1}^{n} D\left(P, A_{j}, A_{j+1}\right)-D\left(P, A_{1}, A_{2}\right)+D\left(P, A_{1}, A_{2}\right) \\
& =\sum_{j=1}^{n} D\left(P, A_{j}, A_{j+1}\right) .
\end{aligned}
$$


Hence by (2.19) we obtain

$$
1 \leq 2 \sum_{j=1}^{n} D\left(P, A_{j}, A_{j+1}\right)+\sum_{j=1}^{n} D\left(P, A_{j}, A_{j+2}\right) .
$$

On the other hand, we have

$$
\sum_{j=1}^{n} D\left(P, A_{j}, A_{j+1}\right) \leq \sum_{j=1}^{n} \sum_{k=1}^{n} D\left(P, A_{j}, A_{k}\right)
$$

and

$$
\sum_{j=1}^{n} D\left(P, A_{j}, A_{j+2}\right) \leq \sum_{j=1}^{n} \sum_{k=1}^{n} D\left(P, A_{j}, A_{k}\right)
$$

Therefore, using (2.20), (2.21), and (2.22), we get that

$$
1 \leq 3 \sum_{j=1}^{n} \sum_{k=1}^{n} D\left(P, A_{j}, A_{k}\right)
$$

Next, taking the sum over $P \in\left\{A_{1}, A_{2}, \ldots, A_{n}\right\}$, we obtain

$$
n \leq 3 \sum_{i=1}^{n} \sum_{j=1}^{n} \sum_{k=1}^{n} D\left(A_{i}, A_{j}, A_{k}\right)
$$

Notice that by (2.5) we have

$$
\sum_{i=1}^{n} \sum_{j=1}^{n} \sum_{k=1}^{n} D\left(A_{i}, A_{j}, A_{k}\right)=6 \sum_{i=1}^{n-2} \sum_{j=i+1}^{n-1} \sum_{k=j+1}^{n} D\left(A_{i}, A_{j}, A_{k}\right)
$$

Combining (2.23) with (2.24), we deduce that

$$
n \leq 18 \sum_{i=1}^{n-2} \sum_{j=i+1}^{n-1} \sum_{k=j+1}^{n} D\left(A_{i}, A_{j}, A_{k}\right)
$$

which yields the desired estimate.

\section{Conclusion}

We obtained new inequalities in the setting of 2-metric spaces and 2-normed linear spaces. Namely, we first derived an analogous version of Theorem 1.1 for 2-metric spaces (see Theorem 2.1). Moreover, we provided a geometric interpretation of our obtained result (see Corollary 2.2). We also presented some interesting consequences following from Theorem 2.1. Next, we considered a problem related to the estimates of areas of triangles and derived a new inequality (see Theorem 2.3). 
Funding

King Saud University.

\section{Abbreviations}

Not applicable.

Availability of data and materials

Not applicable.

Competing interests

The authors declare that they have no competing interests.

Authors' contributions

Both authors contributed equally to the writing of this paper. Both authors read and approved the final manuscript.

\section{Publisher's Note}

Springer Nature remains neutral with regard to jurisdictional claims in published maps and institutional affiliations.

Received: 3 August 2020 Accepted: 12 July 2021 Published online: 28 July 2021

\section{References}

1. Aydi, H., Samet, B.: On some metric inequalities and applications. J. Funct. Spaces 2020, Article ID 3842879 (2020)

2. Brzdęk, J., Ciepliski, K.: On a fixed point theorem in 2-Banach spaces and some of its applications. Acta Math. Sci. 38B(2), 377-390 (2018)

3. Chauhan, S., Imdad, M., Vetro, C.: Unified metrical common fixed point theorems in 2-metric spaces via an implicit relation. J. Oper. 2013, Article ID 186910 (2013)

4. Das, P., Savaş, E., Bhunia, S.: Two valued measure and some new double sequence spaces in 2-normed spaces. Czechoslov. Math. J. 61(3), 809-825 (2011)

5. Ding, Y., Xu, T.-Z.: Approximate solution of generalized inhomogeneous radical quadratic functional equations in 2-Banach spaces. J. Inequal. Appl. 2019, 31 (2019)

6. Dragomir, S.S.: Inequalities for the forward distance in metric spaces. RGMIA Res. Rep. Collect. 23, Article ID 122 (2020)

7. Dragomir, S.S., Goşa, A.C.: An inequality in metric spaces. J. Indones. Math. Soc. 11(1), 33-38 (2005)

8. El-Fassi, I.: Approximate solution of radical quartic functional equation related to additive mapping in 2-Banach spaces. J. Math. Anal. Appl. 455(2), 2001-2013 (2017)

9. Fadail, Z.M., Ahmad, A.B., Ozturk, V., Radenović, S.: Some remarks on fixed point results of b2-metric spaces. Far East J. Math. Sci. 97, 533-548 (2015)

10. Gähler, S.: 2-metrische Räume und ihre topologische Struktur. Math. Nachr. 26, 115-148 (1963)

11. Iseki, K., Sharma, P.L., Sharma, B.K.: Contractive type mapping on 2-metric space. Math. Jpn. 21, 67-70 (1976)

12. Karapinar, E., Noorwali, M.: Dragomir and Gosa type inequalities on b-metric spaces. J. Inequal. Appl. 2019, 29 (2019)

13. Lewandowska, Z.: Linear operators on generalized 2-normed spaces. Bull. Math. Soc. Sci. Math. Roum. 42, 353-368 (1999)

14. Manojlović, V:: On conformally invariant extremal problems. Appl. Anal. Discrete Math. 3, 97-119 (2009)

15. Park, W.G.: Approximate additive mappings in 2-Banach spaces and related topics. J. Math. Anal. Appl. 376(1), 193-202 (2011)

16. Todorcević, V:: Harmonic Quasiconformal Mappings and Hyperbolic Type Metric. Springer, Cham (2019)

17. White, A.: 2-Banach spaces. Math. Nachr. 42, 43-60 (1969)

\section{Submit your manuscript to a SpringerOpen ${ }^{\mathcal{O}}$ journal and benefit from:}

- Convenient online submission

- Rigorous peer review

- Open access: articles freely available online

- High visibility within the field

- Retaining the copyright to your article

Submit your next manuscript at $\boldsymbol{~ s p r i n g e r o p e n . c o m ~}$ 